\title{
Publisher Correction: Development of an immunodeficient pig model allowing long-term accommodation of artificial human vascular tubes
}

\author{
Manabu Itoh', Yosuke Mukae (i) 1, Takahiro Kitsuka1, Kenichi Arai (i) 2, Anna Nakamura², Kazuyoshi Uchihashi², \\ Shuji Toda ${ }^{4}$, Kumika Matsubayashi ${ }^{5}$, Jun-ichi Oyama ${ }^{6}$, Koichi Node (i) ${ }^{6}$, Daisuke Kami ${ }^{7}$, Satoshi Gojo (10 ${ }^{7}$, \\ Shigeki Morita ${ }^{8}$, Takahiro Nishida ${ }^{1}$, Koichi Nakayama ${ }^{2} \&$ Eiji Kobayashi ${ }^{9}$
}

Correction to: Nature Communications https://doi.org/10.1038/s41467-019-10107-1, published online 21 May 2019.

The original version of this Article contained errors in Figs 2 and 3. In Fig. 2b, the scale bar label ' $500 \mu$ m' was originally incorrectly given as ' $200 \mu \mathrm{m}$ '. In Fig. 2 d, the scale bar label ' $500 \mu \mathrm{m}$ ' was originally incorrectly given as ' $100 \mu \mathrm{m}$ '. In Fig. 3c, the scale bar label '200 $\mu \mathrm{m}$ ' was originally incorrectly given as ' $100 \mu \mathrm{m}$ '. This has been corrected in the PDF and HTML versions of the Article.

Published online: 06 August 2019

(i) Open Access This article is licensed under a Creative Commons Attribution 4.0 International License, which permits use, sharing, adaptation, distribution and reproduction in any medium or format, as long as you give appropriate credit to the original author(s) and the source, provide a link to the Creative Commons license, and indicate if changes were made. The images or other third party material in this article are included in the article's Creative Commons license, unless indicated otherwise in a credit line to the material. If material is not included in the article's Creative Commons license and your intended use is not permitted by statutory regulation or exceeds the permitted use, you will need to obtain permission directly from the copyright holder. To view a copy of this license, visit http://creativecommons.org/licenses/by/4.0/.

(C) The Author(s) 2019

\footnotetext{
${ }^{1}$ Department of Thoracic and Cardiovascular Surgery, Faculty of Medicine, Saga University, Saga, Japan. ${ }^{2}$ Department of Regenerative Medicine and Biomedical Engineering, Faculty of Medicine, Saga University, Saga, Japan. ${ }^{3}$ Department of Surgical Pathology, National Hospital Organization Saga Hospital, Saga, Japan. ${ }^{4}$ Department of Pathology \& Microbiology, Faculty of Medicine, Saga University, Saga, Japan. ${ }^{5}$ Cyfuse Biomedical K. K., Tokyo, Japan. ${ }^{6}$ Department of Cardiovascular Medicine, Faculty of Medicine, Saga University, Saga, Japan. ${ }^{7}$ Department of Regenerative Medicine, Kyoto Prefectural University of Medicine, Kyoto, Japan. ${ }^{8}$ Department of Cardiovascular Surgery, National Hospital Organization Kyushu Medical Center, Fukuoka, Japan. ${ }^{9}$ Department of Organ Fabrication, Keio University School of Medicine, Tokyo, Japan. Correspondence and requests for materials should be addressed to K.N. (email: nakayama@me.saga-u.ac.jp) or to E.K. (email: organfabri@a2.keio.jp)
} 